\title{
Derechos fundamentales en tiempos de escasez. A propósito de la Sentencia del TSJ de Galicia 293/2013, de 12 de abroñ ${ }^{1}$
}

\author{
Pablo Nuevo López \\ Profesor Agregado de Derecho Constitucional \\ Universidad Abat Oliba CEU
}

Sumario: Introducción.-1. Los hechos del caso.-2. Los derechos fundamentales en juego y la ponderación.-3. Valoración de la Sentencia.

\section{INTRODUCCIÓN}

Como es bien sabido, la actual crisis económica ha afectado enormemente a las arcas públicas, de modo que las diferentes Administraciones se están viendo obligadas a tomar medidas impopulares tendentes a disminuir el gasto público (los llamados «recortes»). Como resultado de dichas medidas, los ciudadanos se encuentran con que prestaciones que hasta este momento proporcionaban los poderes públicos (por la identificación entre Estado social y Estado prestacional o de bienestar) dejan de estar financiadas públicamente. En este contexto, cuando la prestación objeto de restricciones está conectada con algún derecho fundamental se plantea la cuestión de cómo deben operar las medidas dirigidas al ahorro de caudales públicos en relación con la posición de garante de los derechos fundamentales que, de acuerdo con la Constitución, corresponde a los poderes públicos.

Un ejemplo de ponderación en este contexto lo encontramos en la Sentencia del Tribunal Superior de Justicia de Galicia 293/2013, de 12 de abril, que resuelve el Recurso de Apelación interpuesto por don P.L.B. contra la Sentencia 283/2012, de 10 de agosto, dictada en un procedimiento de protección jurisdiccional de derechos fundamentales por la Ilma. Sra. Magistrado-Juez sustituta del Juzgado de lo Contencioso-Administrativo núm. 1 de Santiago de Compostela, que desestimó el recurso contencioso-administrativo contra la inactividad del Servicio Gallego de Salud consistente en la no dispensación efectiva del medicamento denominado «SOLIRIS $300 \mathrm{mg}$.»

\section{LOS HECHOS DEL CASO}

El demandante, Sr. P.L.B. padece una enfermedad poco común denominada «Hemoglobinuria Paroxística Nocturna». Se trata de una enfermedad rara y de carácter crónico, que consiste en una anemia hemolítica por defecto

${ }^{1}$ Pablo Nuevo López, Profesor Agregado de Derecho Constitucional, Universidad Abat Oliba CEU. 
de la membrana del eritrocito. Como consecuencia de esta enfermedad, los afectados por la misma tienen grave riesgo de trombosis fatales o parésicas.

Esta enfermedad puede ser tratada con la dispensa del principio activo denominado «ECULIZUMAB» (que es el que contiene el medicamento «SOLIRIS $300 \mathrm{mg} . »)$, o bien puede someterse a los afectados por la misma a un trasplante de médula ósea. En caso de recurrirse al tratamiento farmacológico se reduce en un $85 \%$ la posibilidad de que se produzcan eventos trombóticos, equiparándose prácticamente la supervivencia a la población sana. El trasplante de médula ósea requerido, si bien cura la enfermedad, presenta un riesgo estadístico de mortalidad del $30 \%$.

Pues bien, en el caso del Sr. P.L.B. el Servicio Gallego de Salud, a pesar de disponer de sendos informes médicos que aconsejaban la dispensación del principio activo «ECULIZUMAB» (que es el que contiene el medicamento «SOLIRIS $300 \mathrm{mg} . »)$, no autorizó el tratamiento con dicho medicamento.

Según consta en los Antecedentes de Hecho de la Sentencia objeto del presente comentario, en el Expediente administrativo obraban dos Resoluciones administrativas de la Sra. Presidenta de la Comisión Autonómica Central de Farmacia y Terapéutica; una de 1 de agosto de 2011, que señala que el Sr. P.L.B. no cumplía con los requisitos establecidos normativamente para la dispensación del citado medicamento, y otra de 15 de noviembre de 2011, donde se estima el coste del tratamiento en 377.182 euros el primer año y en 360.984 euros el segundo año y siguientes.

A la luz de estos hechos, considera el TSJ de Galicia que debe resolverse procediendo a una ponderación entre los derechos fundamentales en juego y el interés general del ahorro en el gasto público.

\section{LOS DERECHOS FUNDAMENTALES EN JUEGO Y LA PON- DERACIÓN}

Antes de proceder a realizar la ponderación, y toda vez que el recurso había sido promovido por el procedimiento de protección de derechos fundamentales, el TSJ identifica los derechos afectados.

Para ello, el Tribunal recurre a la teoría de la dimensión objetiva de los derechos fundamentales, asumida por tempranamente por nuestro Tribunal Constitucional en su Sentencia 25/19812, vinculando la inactividad del Servi-

${ }^{2}$ Vid. el FJ 5 de la STC 25/1981, de 14 de julio, donde se señala lo siguiente: «En primer lugar, los derechos fundamentales son derechos subjetivos, derechos de los individuos no sólo en cuanto derechos de los ciudadanos en el sentido estricto, sino en cuanto garantizan un "status" jurídico o la libertad en un ámbito de la existencia. Pero al propio tiempo, son elementos esenciales de un ordenamiento objetivo de la comunidad nacional, en cuanto ésta se configura como marco de una convivencia humana justa y pacífica, plasmada históricamente en el Estado de Derecho y, más tarde, en el Estado social de Derecho o el Estado social y democrático de Derecho, según la fórmula de nuestra Constitución (artículo 1.1)». 
cio Gallego de Salud con el derecho a la vida reconocido en el art. 15 CE. Así, considera el TSJ (FJ 4) que el derecho a la vida como fundamento objetivo impone a los poderes públicos el deber de adoptar las medidas necesarias para proteger la vida y la integridad física, deber que se traduce en un derecho subjetivo a recabar amparo judicial frente a toda actuación administrativa, incluso de carácter omisivo ${ }^{3}$. Y es que, prosigue razonando el TSJ haciendo suya la doctrina del TC en su Sentencia 181/2000 (FJ 8),

«el art. 15 de la Constitución no puede ser considerado como un precepto irrelevante a la hora de examinar el régimen legal de la tutela (...) de los bienes de la personalidad que dicho precepto constitucional reconoce y garantiza. La protección constitucional de la vida y de la integridad personal (física y moral) no se reduce al estricto reconocimiento de los derechos subjetivos necesarios para reaccionar jurídicamente frente a las agresiones a ellos inferidas, sino que, además, contiene un mandato de protección suficiente de aquellos bienes de la personalidad, dirigido al legislador y que debe presidir e informar toda su actuación» (FJ 4).

Sentado esto, el TSJ conecta el mandato de protección derivado del derecho a la vida, que convierte al Estado en cierto modo en garante de dicho derecho, con el derecho a la salud, el cual es protegido precisamente mediante la prestación farmacéutica ${ }^{4}$, de acuerdo obviamente con las necesidades de los usuarios establecidas por el personal sanitario competente.

Aunque el TSJ de Galicia no lo explicita, parece que considera que el objeto de la discusión -la dispensación del medicamento adecuado para tratar la «Hemoglobinuria Paroxística Nocturna»- es de una entidad tal que determina que no debamos encuadrar los hechos en el derecho a la protección de la salud (art. $43 \mathrm{CE}$ ), sino que deba considerarse que afecta al derecho a la vida y a la integridad del art. $15 \mathrm{CE}^{5}$.

Es este derecho fundamental reconocido en el art. $15 \mathrm{CE}$ el que somete a ponderación con «la gestión del soporte económico que haga posible su cotidiana consecución, sin perjuicio del deber de todos los poderes públicos de garantizar a todos los ciudadanos el derecho a la protección de la salud» (FJ 6). Para enmarcar la ponderación, el TSJ recurre (FJ 7) a lo establecido por el Tribunal Constitucional en su Auto 239/2012, de 12 de diciembre, conforme al cual:

«Esa ponderación exige colocar de un lado el interés general configurado por el beneficio económico asociado al ahorro vinculado a las medidas adoptadas por el Estado al redefinir el ámbito de los benefi-

${ }^{3}$ Con cita de las SSTC 120/1990, de 27 de junio y 48/1996, de 25 de marzo.

${ }^{4}$ STC 98/2004, de 25 de mayo, FJ 5.

${ }^{5}$ Si no lo hiciera así no sólo debería cambiar el resultado de la ponderación, sino que estaría vedada la utilización del procedimiento preferente y sumario de protección de los derechos fundamentales. 
ciarios del sistema público de salud, y de otro el interés general de preservar el derecho a la salud consagrado en el art. $43 \mathrm{CE}$. Esa contraposición también tiene proyecciones individuales puesto que la garantía del derecho a la salud no sólo tiene una dimensión general asociada a la idea de salvaguarda de la salud pública, sino una dimensión particular conectada con la afectación del derecho a la salud individual de las personas receptoras de las medidas adoptadas por los Gobiernos estatal y autonómico. (...)

Para que este Tribunal valore los intereses vinculados a la garantía del derecho a la salud, es preciso acudir a lo dispuesto en el art. $43 \mathrm{CE}$, en relación con el deber de todos los poderes públicos de garantizar a todos los ciudadanos el derecho a la protección de la salud, cuya tutela les corresponde y ha de ser articulada «a través de medidas preventivas y de las prestaciones y servicios necesarios» (art. 43.1 y $2 \mathrm{CE}$ )» (STC 126/2008, de 27 de octubre, FJ 6). Si, además del mandato constitucional, se tiene en cuenta, como ya lo ha hecho este Tribunal, la vinculación entre el principio rector del art. $43 \mathrm{CE}$ y el art. $15 \mathrm{CE}$ que recoge el derecho fundamental a la vida y a la integridad física y moral, en el sentido de lo reconocido por el Tribunal Europeo de Derechos Humanos (por todos asunto VO c. Francia de 8 de julio de 2004), resulta evidente que los intereses generales y públicos, vinculados a la promoción y garantía del derecho a la salud, son intereses asociados a la defensa de bienes constitucionales particularmente sensibles. (FJ 5)».

De modo que, a juicio del TSJ, «resulta patente la relevancia constitucional-fundamental de la presente controversia contenciosa (...) en la medida en que el derecho a la salud individual desde luego también se integra en el derecho a la vida y a la integridad física y moral de las personas contemplada en el art. 15» (FJ 8), por lo que teniendo en cuenta que no considera acreditado el ahorro que implicaría la no dispensación estimará la pretensión del Sr. P.L.B.

Y es que la mencionada no dispensación del medicamento conlleva, en opinión del TSJ de Galicia «una vulneración mediata del art. 15», al privar de este modo al recurrente de «la única opción terapéutico-medicamentosa autorizada y homologada en el mercado farmacéutico», con la consecuencia de no permitirle tener una calidad de vida normal y asimilable a las de las personas sanas (FJ 12).

Realizada esta ponderación, el TSJ dedica los Fundamentos de Derecho 13 a 25 a argumentar cómo en el caso examinado, además de vulneración del derecho fundamental reconocido en el art. 15CE, había problemas de legalidad, anomalías procedimentales y desviación de poder, los cuales incidían en la vulneración del derecho fundamental mencionado, concluyendo con la estimación del Recurso de Apelación y subsiguientemente revocando la Sentencia 283/2012, de 10 de agosto, del Juzgado de lo Contencioso-Administrativo núm. 1 de Santiago de Compostela. 
En consecuencia, el Tribunal acaba condenando al Servicio Gallego de Salud a la dispensación del tratamiento solicitado (SOLIRIS $300 \mathrm{mg}$ ) durante el tiempo que el personal facultativo oficial considere preciso.

\section{VALORACIÓN DE LA SENTENCIA}

Más allá del caso concreto, en el que no podemos sino alegrarnos de que una persona obtenga del servicio público de salud el tratamiento que necesita para poder llevar una vida razonablemente normal, la Sentencia es susceptible de algunas críticas de fondo en su argumentación.

En primer lugar, es cuando menos discutible el encuadre del caso en el derecho fundamental a la vida y a la integridad reconocido en el art. $15 \mathrm{CE}$. Si bien es cierto que una inadecuada protección de la salud puede devenir una vulneración del derecho a la vida, conectar un caso concreto de prestación sanitaria con el citado derecho y no con el derecho a la protección de la salud del art. 43 exige -a mi entender- un mayor esfuerzo argumentativo que el desplegado por el Tribunal. Esto es, habiendo un derecho específico para el objeto de la discusión, al menos prima facie es ese derecho y no otro el que debe ser considerado como regla, y sólo como excepción -a la luz de las circunstancias: daño efectivo y actual a la salud, pronóstico médico que haga temer por la vida, etc.- se debe recurrir al derecho a la vida del art. 15.

Es verdad que si no hubiera conectado el asunto debatido con el derecho a la vida el Tribunal habría tenido que desestimar la pretensión por inadecuación del procedimiento, pues no se habría podido tramitar el recurso por el preferente y sumario de protección de derechos fundamentales. Pero por lo que señala el Tribunal en los fundamentos 13 a 23 de la Sentencia parece que había motivos más que suficientes para estimar el recurso sin necesidad de entrar en la afección del art. $15 \mathrm{CE}$, aparte de que con ello el Tribunal se habría ahorrado un razonamiento a mi entender de difícil justificación.

Porque, y aquí entramos en la segunda de las cuestiones criticables en la presente Sentencia, el Tribunal realiza la ponderación aplicando doctrina del TC formulada en casos abstractos (conflictos competenciales, como en el Auto 239/2012). En efecto, en dicho Auto estaba en discusión el régimen de las prestaciones sanitarias del sistema nacional de salud en la Comunidad $\mathrm{Au}-$ tónoma del País Vasco, por lo que tenía sentido resolver la ponderación atendiendo al carácter incierto del ahorro que una determinada medida suponía para las arcas públicas (ya que el TC consideró que el Abogado del Estado no había podido acreditar el citado ahorro); de manera que al ponderar el derecho a la protección de la salud (garantizado por una norma) y un ahorro hipotético el TC no tuviera problemas en resolver.

En el caso examinado, en cambio, el Servicio Gallego de Salud sí había cuantificado el ahorro derivado de la no dispensación del medicamento solicitado (377.182 euros el primer año y en 360.984 euros el segundo año y siguientes, según informe del 15 de noviembre de 2011). De ahí que, en mi 
opinión, no cupiera una aplicación «mecánica» de lo señalado en ocasiones anteriores - para casos distintos- el TC.

Al menos, debería haber incluido ese dato en la ponderación, así como haber tomado en cuenta la posibilidad del trasplante de médula ósea, o alguna alternativa diferente.

Y es que la decisión sobre el destino de recursos escasos (siempre, pero sobre todo en situaciones de grave crisis) requiere de una mirada de conjunto de la que, por su propia naturaleza, carece un Tribunal. Es una decisión que corresponde al poder público, que tiene encomendada la tutela de los intereses generales, estando capacitado para ponderar qué concretos tratamientos médicos y farmacológicos quedan cubiertos por el servicio público de salud y cuáles no, precisamente porque tiene capacidad para decidir sobre el conjunto de los gastos públicos, tomando en cuenta la totalidad de los factores.

Cuestión distinta es que, una vez objetiva el poder público su ponderación en una norma señalando los tratamientos a los que tienen derecho los ciudadanos, no pueda la Administración denegarlos con la excusa del ahorro. Pero esto es un problema de legalidad de la actuación administrativa, de fácil resolución sin tener que convertir a los Tribunales en agentes políticos con competencia presupuestaria para establecer las prioridades de gasto para garantizar -señalando el orden- los derechos fundamentales en tiempos de escasez.

RESUMEN: Este artículo analiza la Sentencia del Tribunal Superior de Justicia de Galicia 293/2013, de 12 de abril, en la que se discute la conformidad con los derechos fundamentales de la no dispensación efectiva del medicamento denominado «SOLIRIS $300 \mathrm{mg}$.

PALABRAS CLAVE: Derecho constitucional, crisis económica, medidas de austeridad, sistema de salud, Soliris 300mg.

TITLE: Fundamental rights in times of shortages. Some considerations dealing with the Judgment 293/2013, 12 April (TJS Galicia).

ABSTRACT: This article analyzes the judgment 203/2013, of April 12 of the Superior Court of Galicia (TSJ Galicia) in which the Court deals with the conformity of the non-effective dispensing of a drug called «SOLIRIS $300 \mathrm{mg}$ 》.

KEYWORDS: Constitutional law, economic crisis, austerity measures, health care system, Soliris 300mg.

Recibido: 03.08.2013

Aceptado: 02.09.2013 